\section{Crossing the mucus barrier}

\section{By Tracey Baas, Senior Editor}

A team from The Johns Hopkins University has shown that vaginal delivery of antiviral-loaded, mucus-penetrating particles can help prevent herpes simplex virus infection in mice. ${ }^{1}$ The team thinks the particles have potential utility in infection, cancer, inflammation and other diseases that affect mucosal tissues in the eyes, sinuses, female reproductive tract, respiratory tract and GI tract.

Kala Pharmaceuticals Inc. is the exclusive worldwide licensee of the IP from Johns Hopkins University and is using the technology to develop improved treatments for mucosal tissue diseases, including cystic fibrosis (CF), severe ocular inflammation and ulcerative colitis.

In 2000, the Johns Hopkins group set out to develop nanoparticles that could cross mucus layers and release compounds. To do so, the group based the design of the particles' outside surface on the surfaces of viruses that can penetrate the mucus barrier. The team coated conventional biodegradable nanoparticles with high concentrations of low-molecular weight polyethylene glycol (PEG) and showed that the resulting particles could diffuse through human cervicovaginal mucus (CVM) and also through sputum from the lungs of patients with $\mathrm{CF}^{2-4}$

The unanswered question was whether the mucus-penetrating particles provided broad distribution and long-term retention in vivo in the vagina-two factors that have stymied prior nanoparticle technologies.

The first step was developing an improved mouse model for vaginal delivery studies because both mice and nonhuman primate CVM have different characteristics than human CVM. The team used an estradiolbased treatment to generate mice that produced CVM that more closely resembled the CVM of humans.

"The treatment induces a state that occurs naturally in the mouse estrous cycle in which the vaginal tissue and mucus are similar to those of humans," said Laura Ensign-Hodges, chemical and biomolecular engineering graduate student at Johns Hopkins and lead author of the paper in Science Translational Medicine that described the findings. "We think the mouse model provides a better system to evaluate vaginal delivery than previous mouse models."

"The old mouse model predicted toxicity associated with previous microbicide products that were not evident in humans until Phase III. However, the hormonal treatment used in the old model makes the mucus and the epithelium of the mouse vagina dissimilar from that of the human vagina, so other vitally important characteristics of a vaginal formulation such as distribution, retention and uptake should be studied with the new mouse model," added team member Richard Cone, a professor of biophysics and biology at Johns Hopkins.

In the model, nanoparticles delivered to the vagina formed a continuous layer that coated the entire epithelium, even within deep vaginal folds. Moreover, $60 \%$ of the particles were retained for up to 6 hours. In contrast, conventional nanoparticles aggregated in the luminal mucus and only $10 \%$ were retained for 6 hours.

Particles loaded with a fluorescent compound delivered payload to at least $87 \%$ of the vaginal surface and were retained even after 24 hours, whereas the fluorescent compound formulated in a standard vaginal hydroxyethylcellulose gel delivered payload to $42 \%$ of the vaginal surface.

The researchers also took a close look at whether their nanoparticles caused the vaginal epithelium to secrete cytokines and immune mediators that may enhance susceptibility to sexually transmitted infections-a known potential risk of microbicides such as nonoxynol-9.

In the mice, nonoxynol-9 or conventional nanoparticles both led to an influx of neutrophils, whereas the mucus-penetrating particles did not. In addition, levels of proinflammatory cytokines that are associated with epithelial injury were increased after repeat dosings of nonoxynol-9 but not after repeated dosings of mucus-penetrating particles.

Finally, the researchers wanted to show that the loaded mucuspenetrating particles could prevent herpes simplex virus (HSV) infection in mice. Animals pretreated with nanoparticles loaded with the generic HSV drug acyclovir showed 53\% protection, whereas mice pretreated with soluble acyclovir showed $12 \%$ protection.

Thomas Moench, president and COO of ReProtect Inc., said acyclovir was a good choice for the study because it has notoriously poor efficacy in the prophylactic setting when given in solution. The next step, he said, should be to test the nanoparticles with a more potent drug.

Team leader Justin Hanes agreed. "Our focus with this work was to show that mucus-penetrating particles enhanced efficacy and did not cause toxicity or inflammation that could enhance virus infection. In this study, we weren't looking for perfect or complete protection," he said. "We specifically selected acyclovir because it has poor efficacy even though it is the best available. This way we could test whether the particles improved antiviral efficacy."

Hanes is professor of ophthalmology in the Wilmer Eye Institute at Johns Hopkins and director of the Center for
"Our focus with this work was to show that mucus-penetrating particles enhanced efficacy and did not cause toxicity or inflammation that could enhance virus infection." - Justin Hanes,

The Johns Hopkins University Nanomedicine at The Johns Hopkins University School of Medicine. $\mathrm{He}$ is a cofounder and director of Kala.

\section{Eyeing indications}

Kevin Pojasek, VP of corporate development at Kala, said the company is using its mucosa-penetrating platform to engineer medicines for treating diseases affecting mucosal tissues. Kala's internal programs 


\section{ANALYSIS}

are in preclinical development, and the company also will seek deals to apply the technology platform to partners' compounds.

Meanwhile, Hanes first wants to test nanoparticles loaded with Viread tenofovir in a mouse model of HIV. The Hopkins team also is developing mucus-penetrating nanoparticles to deliver chemotherapeutics for lung and cervical cancers, anti-inflammatories for lung inflammation and chronic rhinosinusitis, microbicides for rectal use and gene therapy for $\mathrm{CF}$.

Gilead Sciences Inc. markets the reverse transcriptase inhibitor Viread to treat HIV infection. The company is running a Phase IIb trial of tenofovir $1 \%$ vaginal gel to prevent HIV infection.

Hanes previously collaborated with Copernicus Therapeutics Inc. to develop DNA-carrying nanoparticles to deliver gene therapy for CF and ophthalmic disease.

"The nanoparticle formulation consists of single molecules of DNA compacted with polyethylene glycol-substituted lysine peptides," said Mark Cooper, SVP of science and medical affairs at Copernicus. "Additional nanoparticle refinements, such as optimization of PEG size and density, permit DNA nanoparticle diffusion through mucus, as pioneered by Hanes. The small size of our particles permits transit through the nuclear membrane pore, allowing delivery of therapeutic genes."

Copernicus' nanoparticle-delivered gene therapy to treat CF is in Phase I/II testing.
Johns Hopkins holds a European patent covering the work reported in Science Translational Medicine and has filed for a patent in the U.S. The IP is licensed to Kala.

Baas, T. SciBX 5(27); doi:10.1038/scibx.2012.697

Published online July 12, 2012

\section{REFERENCES}

1. Ensign, L.M. et al. Sci. Transl. Med.; published online June 13, 2012; doi:10.1126/scitransImed.3003453

Contact: Justin Hanes, The Johns Hopkins University School of Medicine, Baltimore, Md.

e-mail: hanes@jhu.edu

2. Wang, Y.-Y. et al. Angew. Chem. Int. Ed. 47, 9726-9729 (2008)

3. Lai, S.K. et al. Proc. Natl. Acad. Sci. USA 104, 1482-1487 (2007)

4. Tang, B.C. et al. Proc. Natl. Acad. Sci. USA 106, 19268-19273 (2009)

\section{COMPANIES AND INSTITUTIONS MENTIONED}

Copernicus Therapeutics Inc., Cleveland, Ohio Gilead Sciences Inc. (NASDAQ:GILD), Foster City, Calif. The Johns Hopkins University, Baltimore, Md. The Johns Hopkins University School of Medicine, Baltimore, Md.

Kala Pharmaceuticals Inc., Waltham, Mass.

ReProtect Inc., Baltimore, Md. 